\title{
Centroid-based methods for calculating quantum reaction rate constants: Centroid sampling versus centroid dynamics
}

\author{
Qiang Shi and Eitan Geva \\ Department of Chemistry, University of Michigan, Ann Arbor, Michigan 48109-1055
}

(Received 5 October 2001; accepted 30 November 2001)

\begin{abstract}
A new method was recently introduced for calculating quantum mechanical rate constants from centroid molecular dynamics (CMD) simulations [E. Geva, Q. Shi, and G. A. Voth, J. Chem. Phys. 115, 9209 (2001)]. This new method is based on a formulation of the reaction rate constant in terms of the position-flux correlation function, which can be approximated in a well defined way via CMD. In the present paper, we consider two different approximated versions of this new method, which enhance its computational feasibility. The first approximation is based on propagating initial states which are sampled from the initial centroid distribution, on the classical potential surface. The second approximation is equivalent to a classical-like calculation of the reaction rate constant on the centroid potential, and has two distinct advantages: (1) it bypasses the problem of inefficient sampling which limits the applicability of the full CMD method at very low temperatures; (2) it has a well defined TST limit which is directly related to path-integral quantum transition state theory (PI-QTST). The approximations are tested on a model consisting of a symmetric double-well bilinearly coupled to a harmonic bath. Both approximations are quite successful in reproducing the results obtained via full CMD, and the second approximation is shown to provide a good estimate to the exact high-friction rate constants at very low temperatures. (C) 2002 American Institute of Physics. [DOI: 10.1063/1.1445120]
\end{abstract}

\section{INTRODUCTION}

The calculation of solution-phase quantum mechanical reaction rate constants in anharmonic systems represent an ongoing challenge for theoretical and computational chemistry. ${ }^{1}$ Most previous attempts to address this challenge were based on one of the following approaches:

(a) The quantum transition state theory (QTST) approach. ${ }^{2-13}$ Quantum mechanical expressions for reaction rate constants that are based on equilibrium thermodynamic averages are termed QTST. One of the most successful formulations of QTST is path-integral QTST (PI-QTST). ${ }^{3-6,8,9,12}$ According to the path-integral formulation of quantum mechanics, ${ }^{14,15}$ the equilibrium dynamics of a quantum particle is analogous to that of a classical cyclic chain of beads connected by harmonic springs. ${ }^{16,17}$ The center-of-mass of such a chain is known as its centroid. The structure of PI-QTST is similar to that of classical TST, ${ }^{18}$ except that the classical positions are replaced by the centroids of the corresponding chains. Several suggestions have also been made for introducing dynamical corrections to rate constants calculated via QTST.6,19,20

(b) The semiclassical approach. This approach is based on the reactive-flux formulation of the reaction rate constant in terms of the flux-heaviside or flux-flux correlation functions. ${ }^{2,21,22}$ In this case one uses a semiclassical approximation in order to estimate the corresponding quantum correlation functions. ${ }^{23-28}$

(c) The analytical continuation approach. ${ }^{29-34}$ The evaluation of imaginary time quantum mechanical correlation functions is computationally feasible for relatively complex systems via Monte Carlo or molecular dynamics simulations on the corresponding classical chains (PIMC and PIMD, respectively). ${ }^{16,17}$ In this case, one attempts to analytically continue the imaginary time quantum mechanical fluxheaviside correlation function to real time. ${ }^{29,34,35}$

In a recent paper, we introduced a new and potentially powerful approach for the calculation of quantum reaction rate constants. ${ }^{36}$ Like PI-QTST, it is based on the centroid concept. However, it avoids any kind of TST-like approximations, and explicitly accounts for dynamical effects within the framework of centroid molecular dynamics (CMD). CMD is an approximate method for calculating real-time quantum correlation functions. ${ }^{9,37-43}$ It is based on the hypothesis that the centroid follows classical-like dynamics, and that quantum effects can be incorporated by modifying the force fields, as well as by representing dynamical observables in terms of suitably defined "centroid symbols." The new method has been systematically tested ${ }^{36}$ on a benchmark system consisting of a double-well potential bilinearly coupled to a harmonic bath, ${ }^{44}$ and was found to provide an excellent approximation for the exact rate constant on a wide range of temperatures and frictions. ${ }^{36} \mathrm{At}$ the same time, the new method is subject to two practical limitations:

(1) Generally speaking, CMD simulation of many-body systems with anharmonic interaction potentials requires an on-the-fly determination of the centroid force at every time step. Although the computational effort is often feasible, ${ }^{45-54}$ it is still very demanding and therefore prohibitive if one wishes to explore the parameter space of a problem.

(2) Quantum delocalization renders centroid sampling of the 
initial state inefficient at low temperatures, thereby making it increasingly more difficult to perform the calculation under these conditions (see Sec. IV). ${ }^{36}$

In the present paper we address these difficulties by considering two different approximated versions of the new method which are computationally more economical.

The remainder of this paper is organized in the following way: A short overview of the new method is given in Sec. II. The first approximation, which is based on propagating initial states sampled from the initial centroid distribution, on the classical potential surface, is discussed in Sec. III. The second approximation which is equivalent to using the reactive-flux formulation on a centroid potential is discussed in Sec. IV. A similar approximation was previously suggested by Schenter et al. as a way of introducing dynamical corrections to PI-QTST. ${ }^{20}$ In Sec. IV, we derive this approximation from the CMD expression in the case of the harmonic bath, and critically examine several simplified versions of it. The main conclusions of this work are summarized in Sec. V.

\section{REACTION RATE CONSTANTS FROM CMD SIMULATIONS}

\section{A. General formalism}

In this section we give a brief overview of our recently proposed method for calculating reaction rate constants from CMD simulations. ${ }^{36}$ To this end, consider a unimolecular reaction, such as isomerization, that takes place in solution, along a predefined reaction coordinate. The total Hamiltonian is given by

$$
\hat{H}=\frac{\hat{p}^{2}}{2 m}+\sum_{i=1}^{N} \frac{\left(\hat{P}^{(i)}\right)^{2}}{2 M^{(i)}}+V(\hat{\mathbf{Q}}, \hat{s}) .
$$

Here, as in the rest of this paper, we use boldface letters for vectors and letters capped with a $\wedge$, e.g., $\hat{A}$, for operators. $\hat{s}$, $\hat{p}$, and $m$ are the reaction coordinate, conjugate momentum, and corresponding mass, respectively; $\hat{\mathbf{Q}}=\left(\hat{Q}^{(1)}, \ldots, \hat{Q}^{(N)}\right)$, $\hat{\mathbf{P}}=\left(\hat{P}^{(1)}, \ldots, \hat{P}^{(N)}\right)$, and $\left\{M^{(i)}\right\}$ are the coordinates, conjugate momenta, and masses of the bath degrees of freedom, respectively; and $V(\hat{s}, \hat{\mathbf{Q}})$ is the total potential energy that includes the potential energy along the bare reaction coordinate, the potential energy of the bare bath, and the interaction potential between the reaction coordinate and the bath. It is assumed that the potential along the bare reaction coordinate has the shape of a double-well, and that the barrier top is located at $s=0$.
The reactive system is said to be in the reactant state if $s<0$ and in the product state if $s>0 . P_{R}$ and $P_{P}$ are defined as the mole fractions of the reactant and the product, respectively,

$$
P_{P}=1-P_{R}=\langle h(\hat{s})\rangle \equiv\langle\hat{h}\rangle,
$$

where $h(\hat{s})$ is the Heaviside function operator $\left[\left\langle s|h(\hat{s})| s^{\prime}\right\rangle\right.$ $=\delta\left(s-s^{\prime}\right)$ for $s>0$, and zero otherwise $]$. The reaction rate constant, $k$ is defined by

$$
\dot{P}_{P}=-\dot{P}_{R}=-k_{R P} P_{P}+k_{P R} P_{R},
$$

or equivalently,

$$
\delta \dot{P}_{i}=-k \delta P_{i}
$$

where $i=P$ or $R, k=k_{P R}+k_{R P}, \delta P_{i}=P_{i}-P_{i}^{\mathrm{eq}}, P_{P}^{\mathrm{eq}}=k_{P R} /$ $k$, and $P_{R}^{\mathrm{eq}}=k_{R P} / k$.

As was shown in Ref. 36, the exact quantum reaction rate constant may be expressed in terms of the Kubotransformed position-flux correlation function,

$$
k=-\frac{C_{\hat{s}, \hat{F}}^{\text {Kubo }}(t)}{C_{\delta \hat{s}, \delta \hat{h}}^{\text {Kubo }}(0)} .
$$

Here, $\quad \delta \hat{A}(\tau)=\hat{A}(\tau)-\langle\hat{A}\rangle_{\mathrm{eq}}, \quad\langle\hat{A}\rangle_{\mathrm{eq}}=\operatorname{Tr}\left[e^{-\beta \hat{H}} \hat{A}\right] / Z, \quad Z$ $=\operatorname{Tr}\left[e^{-\beta \hat{H}}\right], \hat{A}(\tau)=e^{i \hat{H} \tau / \hbar} \hat{A} e^{-i \hat{H} \tau / \hbar}$,

$$
\begin{aligned}
C_{\hat{A} \hat{B}}^{\text {Kubo }}(t)= & \frac{1}{\beta Z} \int_{0}^{\beta} d \lambda \operatorname{Tr}\left\{e^{-(\beta-\lambda) \hat{H}}\right. \\
& \left.\times \hat{A} e^{-\lambda \hat{H}} e^{i \hat{H} t / \hbar} \hat{B} e^{-i \hat{H} t / \hbar}\right\} .
\end{aligned}
$$

is the quantum Kubo transformed correlation function, and

$$
\hat{F}=d \hat{h} / d t=i[\hat{H}, h(\hat{s})] / \hbar=\frac{1}{2 m}[\hat{p} \delta(\hat{s})+\delta(\hat{s}) \hat{p}]
$$

is the flux operator.

The expression for the reaction rate constant in Eq. (5) is particularly advantageous from the viewpoint of CMD. This is because the latter can provide a well-defined approximation for correlation functions involving at least one operator which is linear in the position (or momentum) operators. ${ }^{42,43}$ The CMD approximation of the quantum reaction rate constant in Eq. (5) is given by ${ }^{36}$

$$
k \approx k_{\mathrm{CMD}}=\frac{\int d s_{c} \int d p_{c} \int d \mathbf{Q}_{\mathbf{c}} \int d \mathbf{P}_{\mathbf{c}} \rho_{c}\left(s_{c}, p_{c}, \mathbf{Q}_{\mathbf{c}}, \mathbf{P}_{\mathbf{c}}\right) s_{c}(-t) F_{c}\left(s_{c}, p_{c}, \mathbf{Q}_{\mathbf{c}}\right)}{\int d s_{c} \int d p_{c} \int d \mathbf{Q}_{\mathbf{c}} \int d \mathbf{P}_{\mathbf{c}} \rho_{c}\left(s_{c}, p_{c}, \mathbf{Q}_{\mathbf{c}}, \mathbf{P}_{\mathbf{c}}\right) \delta s_{c} \delta h_{c}\left(s_{c}, \mathbf{Q}_{\mathbf{c}}\right)} .
$$


It should be noted that $k$ and approximations to it are expected to be explicitly time-dependent during an initial short transient period, $0<t<t_{p}(\ll 1 / k)$, in the following which they reach the "plateau region," where they acquire a fixed value. $^{55,56}$

Other quantities that appear in Eq. (8) have the following form:

$\boldsymbol{\rho}_{c}\left(s_{c}, p_{c}, \mathbf{Q}_{\mathbf{c}}, \mathbf{P}_{\mathbf{c}}\right)=\boldsymbol{\rho}_{c}\left(s_{c}, \mathbf{Q}_{\mathbf{c}}\right) e^{-\beta\left[\Sigma_{i=1, N}\left[\left(P_{c}^{(i)}\right)^{2} / 2 M^{(i)}\right]+\left(p_{c}^{2} / 2 m\right)\right]}$,

$h_{c}$ and $F_{c}$ are the centroid symbols of the Heaviside and flux operators, and are given by

$$
\begin{aligned}
& h_{c}\left(s_{c}, \mathbf{Q}_{\mathbf{c}}\right)=\frac{\rho_{c}^{+}\left(s_{c}, \mathbf{Q}_{\mathbf{c}}\right)}{\rho_{c}\left(s_{c}, \mathbf{Q}_{\mathbf{c}}\right)}, \\
& F_{c}\left(s_{c}, p_{c}, \mathbf{Q}_{\mathbf{c}}\right)=\frac{p_{c}}{m} \frac{\rho_{c}^{\prime}\left(s_{c}, \mathbf{Q}_{\mathbf{c}}\right)}{\rho_{c}\left(s_{c}, \mathbf{Q}_{\mathbf{c}}\right)},
\end{aligned}
$$

where

$$
\begin{aligned}
& \rho_{c}\left(s_{c}, \mathbf{Q}_{\mathbf{c}}\right)=\mathcal{C} \int_{s(0)=s(\beta \hbar)} D s(\tau) \int_{\mathbf{Q}(0)=\mathbf{Q}(\beta \hbar)} D \mathbf{Q}(\tau) \\
& \times \delta\left[s_{c}-(\beta \hbar)^{-1} \int_{0}^{\beta \hbar} d \tau s(\tau)\right] \delta\left[\mathbf{Q}_{\mathbf{c}}-(\beta \hbar)^{-1}\right. \\
& \left.\times \int_{0}^{\beta \hbar} d \tau \mathbf{Q}(\tau)\right] \exp \{-\mathcal{S}[s(\tau), \mathbf{Q}(\tau)] / \hbar\} \\
& =\lim _{P \rightarrow \infty} \mathcal{N}(P) \int d s_{1} \cdots \int d s_{P} \int d \mathbf{Q}_{1} \cdots \int d \mathbf{Q}_{\mathbf{P}} \\
& \times \delta\left[s_{c}-\frac{1}{P} \sum_{k=1}^{P} s_{k}\right] \delta\left[\mathbf{Q}_{\mathbf{c}}-\frac{1}{P} \sum_{k=1}^{P} \mathbf{Q}_{\mathbf{k}}\right] \\
& \times \exp \left\{-\mathcal{S}\left(s_{1}, \ldots, s_{P}, \mathbf{Q}_{\mathbf{1}}, \ldots, \mathbf{Q}_{\mathbf{P}}\right) / \hbar\right\}, \\
& \rho_{c}^{+}\left(s_{c}, \mathbf{Q}_{\mathbf{c}}\right)=\mathcal{C} \int_{s(0)=s(\beta \hbar)} D s(\tau) \\
& \times \int_{\mathbf{Q}(0)=\mathbf{Q}(\beta \hbar)} D \mathbf{Q}(\tau)\left[(\beta \hbar)^{-1} \int_{0}^{\beta \hbar} d \tau h(s(\tau))\right] \\
& \times \delta\left[s_{c}-(\beta \hbar)^{-1} \int_{0}^{\beta \hbar} d \tau s(\tau)\right] \delta\left[\mathbf{Q}_{\mathbf{c}}-(\beta \hbar)^{-1}\right. \\
& \left.\times \int_{0}^{\beta \hbar} d \tau \mathbf{Q}(\tau)\right] \exp \{-\mathcal{S}[s(\tau), \mathbf{Q}(\tau)] / \hbar\} \\
& =\lim _{P \rightarrow \infty} \mathcal{N}(P) \int d s_{1} \cdots \int d s_{P} \int d \mathbf{Q}_{1} \cdots \\
& \times \int d \mathbf{Q}_{\mathbf{P}}\left[\frac{1}{P} \sum_{k=1}^{P} h\left(s_{k}\right)\right] \\
& \times \delta\left[s_{c}-\frac{1}{P} \sum_{k=1}^{P} s_{k}\right] \delta\left[\mathbf{Q}_{\mathbf{c}}-\frac{1}{P} \sum_{k=1}^{P} \mathbf{Q}_{\mathbf{k}}\right] \\
& \times \exp \left\{-\mathcal{S}\left(s_{1}, \ldots, s_{P}, \mathbf{Q}_{\mathbf{1}}, \ldots, \mathbf{Q}_{\mathbf{P}}\right) / \hbar\right\},
\end{aligned}
$$

$$
\begin{gathered}
\frac{1}{\hbar} \mathcal{S}\left[s_{1}, \ldots, s_{P}, \mathbf{Q}_{\mathbf{1}}, \ldots, \mathbf{Q}_{\mathbf{P}}\right] \\
=\beta \sum_{k=1}^{P}\left\{\frac{1}{2} m \omega_{P}^{2}\left(s_{k}-s_{k+1}\right)^{2}\right. \\
\left.\quad+\sum_{i=1}^{N} \frac{1}{2} M^{(i)} \omega_{P}^{2}\left(Q_{k}^{(i)}-Q_{k+1}^{(i)}\right)^{2}+\frac{1}{P} V\left(s_{k}, \mathbf{Q}_{k}\right)\right\}, \\
\mathcal{C}=\left\{\left(\frac{2 \pi \beta \hbar^{2}}{m}\right) \prod_{i=1}^{N}\left(\frac{2 \pi \beta \hbar^{2}}{M^{(i)}}\right)\right\}^{1 / 2}, \\
\mathcal{N}(P)=\mathcal{C}\left\{\left(\frac{m P}{2 \pi \beta \hbar^{2}}\right) \prod_{i=1}^{N}\left(\frac{M^{(i)} P}{2 \pi \beta \hbar^{2}}\right)\right\}^{P / 2},
\end{gathered}
$$$$
\text { and } \omega_{P}^{2}=P /(\beta \hbar)^{2} \text {. }
$$

It should be emphasized that within the CMD approximation, $s_{c}(-t)$ in Eq. (8) is obtained by propagating $s_{c}$ backwards in time as a classical position, but on the centroid potential, $V_{c m}\left(\mathbf{Q}_{\mathbf{c}}, s_{c}\right)$, which is defined by

$$
\rho_{c}\left(\mathbf{Q}_{\mathbf{c}}, s_{c}\right) \equiv e^{-\beta V_{c m}\left(\mathbf{Q}_{\mathbf{c}}, s_{c}\right)},
$$


and which is distinctly different from the corresponding classical potential, $V(\mathbf{Q}, s)$. It should also be noted that Eq. (8) involves the following approximation regarding the dynamics of the flux centroid symbol: $:^{36,42}$

$$
F_{c}\left(s_{c}, p_{c}, \mathbf{Q}_{\mathbf{c}} ; t\right) \approx F_{c}\left(s_{c}(t), p_{c}(t), \mathbf{Q}_{\mathbf{c}}(t)\right),
$$

where $s_{c}(t), \mathbf{Q}_{\mathbf{c}}(t), \mathbf{P}_{\mathbf{c}}(t)$ are propagated as classical positions and momenta on the centroid potential surface, $V_{c m}\left(s_{c}, \mathbf{Q}_{\mathbf{c}}\right)$.

\section{B. Application to a system bilinearly coupled to a harmonic bath}

It is important to test any approximation for the quantum mechanical rate constant on a multidimensional model system for which exact solutions are available. The benchmark used in the present work is based on a symmetric doublewell potential bilinearly coupled to a harmonic bath, for which the exact quantum reaction rate constants have been calculated by Topaler and Makri. ${ }^{44}$ For this model, the total Hamiltonian is given by

$$
\begin{aligned}
\hat{H}= & \frac{\hat{p}^{2}}{2 m}+V_{0}(\hat{s})+\sum_{j}\left[\frac{\left(\hat{P}^{(j)}\right)^{2}}{2 M^{(j)}}+\frac{1}{2} M^{(j)}\left(\omega^{(j)}\right)^{2}\right. \\
& \left.\times\left(\hat{Q}^{(j)}-\frac{c^{(j)} \hat{s}}{M^{(j)}\left(\omega^{(j)}\right)^{2}}\right)^{2}\right]
\end{aligned}
$$

where

$$
V_{0}(s)=-a_{1} s^{2}+a_{2} s^{4} .
$$

The spectral density of the bath is assumed to be Ohmic with an exponential cutoff,

$$
J(\omega)=\frac{\pi}{2} \sum_{j} \frac{\left(c^{(j)}\right)^{2}}{M^{(j)} \omega^{(j)}} \delta\left(\omega-\omega^{(j)}\right)=\eta \omega e^{-\omega / \omega_{c}},
$$

and $m$ is taken to be the mass of a proton. The parameters for $V_{0}(s)$ and $J(\omega)$ are identical to these used for the DW1 model in Ref. 44. It should be noted that Eq. (8) was tested on the same model in Ref. 36 and $k_{\mathrm{CMD}}$ was found to be in good agreement with the exact results for a wide range of temperatures and frictions.

Another advantage of the above mentioned model system is that in this case, the average over the harmonic bath modes can be performed analytically. This leads to the following expression for the centroid distribution for the overall system: ${ }^{36}$

$$
\rho_{c}\left(s_{c}, \mathbf{Q}_{\mathbf{c}}\right)
$$

$$
=\mathcal{A}(P) e^{-\beta\left[V_{\mathrm{eff}}\left(s_{c}\right)+\Sigma_{j=1}^{N} \frac{1}{2} M^{(j)}\left(\omega^{(j)}\right)^{2}\left(Q_{c}^{(j)}-\left[c^{(j)} s_{c} / M^{(j)}\left(\omega^{(j)}\right)^{2}\right]\right)^{2}\right]} .
$$

The explicit expressions for $\mathcal{A}(P)$ and $V_{\text {eff }}\left(s_{c}\right)$ were given in Ref. 36. For our purposes here, it is sufficient to note that $V_{\text {eff }}\left(s_{c}\right)$ is a function of $s_{c}$ only, and is shifted relative to the bare potential, $V_{0}\left(s_{c}\right)$, by a bath-induced term. It is also important to note that $\rho_{c}^{\prime}\left(s_{c}, \mathbf{Q}_{\mathbf{c}}\right)$ and $\rho_{c}^{+}\left(s_{c}, \mathbf{Q}_{\mathbf{c}}\right)$ are given by expressions similar to Eq. (22), except that $V_{\text {eff }}\left(s_{c}\right)$ is replaced by appropriately defined $V_{\text {eff }}^{\prime}\left(s_{c}\right)$ and $V_{\text {eff }}^{+}\left(s_{c}\right)$, respec-

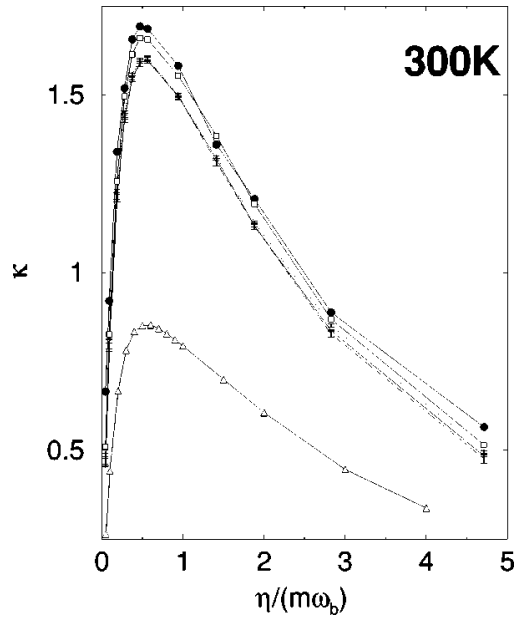

FIG. 1. The transmission coefficient as a function of friction, for DW1 at $300 \mathrm{~K}$. Shown are the exact quantum results (solid line with filled circles), the classical results (solid line with opaque triangles), the results obtained from the $k_{\mathrm{CMD}}$ approximation (solid line), the $k_{\mathrm{CeS}}$ approximation (dashed line), the $k_{\mathrm{ClS}}$ approximation (dotted line), and the $k_{\mathrm{CIS}}^{c}$ approximation (solid line with opaque squares)

tively (cf. Ref. 36 for details). An important outcome of this is that the centroid symbols of the flux and Heaviside operators, $F_{c}$ and $h_{c}$, respectively, become independent of the bath coordinates [cf. Eq. (10)].

Finally, we note that following Ref. 44, the results of the calculations are presented below in terms of the transmission coefficient,

$$
\kappa=k / k_{\mathrm{cl}}^{\mathrm{TST}}
$$

where

$$
k_{\mathrm{cl}}^{\mathrm{TST}}=\frac{1}{m} \frac{\langle\delta(s) p h(p)\rangle}{\langle 1-h(s)\rangle}
$$

is the classical TST reaction rate constant $(\langle\cdots\rangle)$ corresponds to averaging over the classical many-body Boltzmann distribution).

The transmission coefficients obtained for this model from Eq. (8), at $300 \mathrm{~K}, 200 \mathrm{~K}$, and $100 \mathrm{~K}$, are represented by solid lines in Figs. 1, 2, and 3, respectively, for a wide range of frictions. As can be seen, $k_{\mathrm{CMD}}$ provides a good approximation to the exact quantum results, which are represented by a solid lines with filled circles, and capture much of the quantum enhancement relative to the corresponding classical results, which are represented by a solid line with opaque triangles.

\section{CENTROID SAMPLING AND CLASSICAL DYNAMICS}

The centroid symbol of the flux operator, Eq. (10), deserves special attention. In the classical limit, $\rho_{c}^{\prime}\left(s_{c}, \mathbf{Q}_{\mathbf{c}}\right) / \rho_{c}\left(s_{c}, \mathbf{Q}_{\mathbf{c}}\right)$ is replaced by $\delta\left(s_{c}\right)$, such that only trajectories that start at the barrier top, $s_{c}=0$, are sampled. However, moving away from the classical limits turns $\rho_{c}^{\prime}\left(s_{c}, \mathbf{Q}_{\mathfrak{c}}\right) / \rho_{c}\left(s_{c}, \mathbf{Q}_{\mathbf{c}}\right)$ into an increasingly wider distribution of the initial values of $s_{c}$. The width of this distribution 


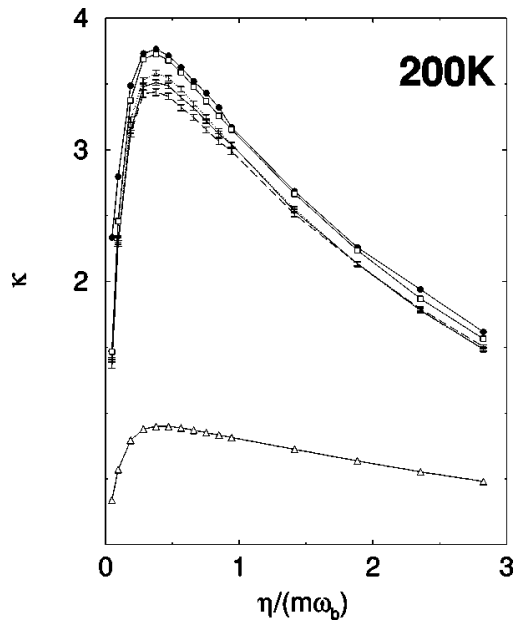

FIG. 2. The transmission coefficient as a function of friction, for DW1 at $200 \mathrm{~K}$. Shown are the exact quantum results (solid line with filled circles), the classical results (solid line with opaque triangles), the results obtained from the $k_{\mathrm{CMD}}$ approximation (solid line), the $k_{\mathrm{CeS}}$ approximation (dashed line), the $k_{\mathrm{CIS}}$ approximation (dotted line), and the $k_{\mathrm{CIS}}^{c}$ approximation (solid line with opaque squares).

increases with the quantum nature of the problem, and can therefore be viewed as a manifestation of quantum delocalization. Another useful perspective is based on the realization that $\rho_{c}^{\prime}\left(s_{c}, \mathbf{Q}_{\mathbf{c}}\right)$ and $\rho_{c}\left(s_{c}, \mathbf{Q}_{\mathbf{c}}\right)$ are analogous to partition functions of a system consisting of $N+1$ cyclic polymers whose centroids are fixed at $\left(s_{c}, Q_{c}^{(1)}, \ldots, Q_{c}^{(N)}\right)$. While this is the only constraint in the case of $\rho_{c}\left(s_{c}, \mathbf{Q}_{\mathbf{c}}\right), \rho_{c}^{\prime}\left(s_{c}, \mathbf{Q}_{\mathbf{c}}\right)$ includes another constraint, namely, that the position of one of the beads in the $s_{c}$-centered chain is fixed at the barrier top position, i.e., at $s=0$. The width of $\rho_{c}^{\prime}\left(s_{c}, \mathbf{Q}_{\mathbf{c}}\right) / \rho_{c}\left(s_{c}, \mathbf{Q}_{\mathbf{c}}\right)$ as a function of $s_{c}$ therefore depends on the typical extension of a cyclic polymer which is attached to the barrier top, and is expected to increase as the masses and temperatures decrease.

Substituting $F_{c}$ from Eq. (10) into Eq. (8) and canceling out $\rho_{c}\left(s_{c}, \mathbf{Q}_{\mathbf{c}}\right)[$ cf. Eq. (9)], the centroid flux-position correlation function can be put in the following form:

$$
\begin{aligned}
& \int d s_{c} \int d p_{c} \int d \mathbf{Q}_{\mathbf{c}} \int d \mathbf{P}_{\mathbf{c}} \rho_{c}\left(s_{c}, p_{c}, \mathbf{Q}_{\mathbf{c}}, \mathbf{P}_{\mathbf{c}}\right) s_{c}(-t) \\
& \times F_{c}\left(s_{c}, p_{c}, \mathbf{Q}_{\mathbf{c}}\right) \\
& =\int d s_{c} \int d p_{c} \int d \mathbf{Q}_{\mathbf{c}} \int d \mathbf{P}_{\mathbf{c}} \boldsymbol{\rho}_{c}^{\prime}\left(s_{c}, \mathbf{Q}_{\mathbf{c}}\right) \\
& \times e^{-\beta\left[\Sigma_{i=1, N}\left[\left(P_{c}^{(i)}\right)^{2} / 2 M^{(i)}\right]+\left(p_{c}^{2} / 2 m\right)\right]} s_{c}(-t) \frac{p_{c}}{m} .
\end{aligned}
$$

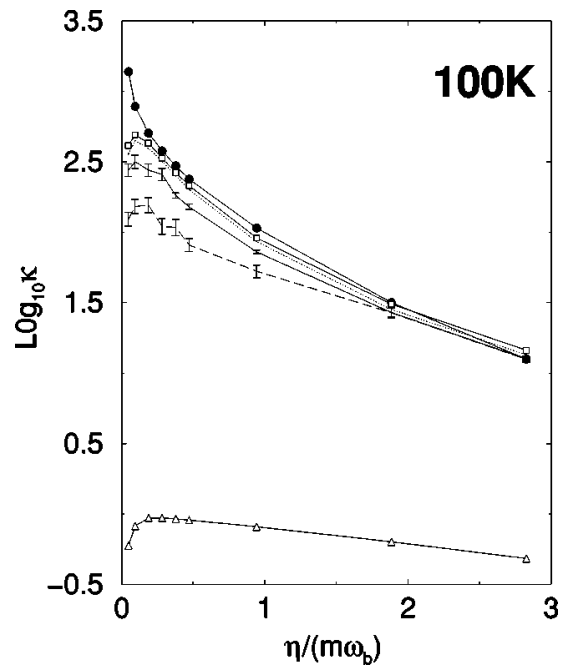

FIG. 3. The transmission coefficient as a function of friction, for DW1 at $100 \mathrm{~K}$. Shown are the exact quantum results (solid line with filled circles), the classical results (solid line with opaque triangles), the results obtained from the $k_{\mathrm{CMD}}$ approximation (solid line), the $k_{\mathrm{CeS}}$ approximation (dashed line), the $k_{\mathrm{CIS}}$ approximation (dotted line), and the $k_{\mathrm{CIS}}^{c}$ approximation (solid line with opaque squares). Note that $\log _{10}(\kappa)$, rather than $\kappa$, is plotted.

Quantum corrections are introduced into Eq. (25) in two distinctively different ways:

(1) The initial distribution of $s_{c}$ is dictated by $\rho_{c}^{\prime}\left(s_{c}, \mathbf{Q}_{\mathbf{c}}\right)$ rather than by $\delta\left(s_{c}\right) e^{-V\left(s_{c}, \mathbf{Q}_{\mathbf{c}}\right)}$.

(2) The dynamics of $s_{c}$ is governed by the centroid potential, $V_{c m}\left(s_{c}, \mathbf{Q}_{\mathbf{c}}\right)$, rather than the classical potential, $V\left(s_{c}, \mathbf{Q}_{\mathbf{c}}\right)$.

It is natural to ask whether one of the two quantum effects plays a more dominant role than the other? The answer to this question has important practical implications because initial centroid sampling only requires a single PIMD/PIMC simulation at $t=0$, whereas centroid dynamics requires that a PIMD/PIMC simulation is performed at every time step if the centroid force is to be evaluated onthe-fly.

We start by examining this question in the context of our model system, namely, a symmetric double-well potential bilinearly coupled to a harmonic bath (cf. Sec. II B). The centroid dynamics component of the CMD calculation can be suppressed by sampling the initial values of $s_{c}$ from the nonclassical centroid distribution, $\rho_{c}^{\prime}\left(s_{c}, \mathbf{Q}_{\mathbf{c}}\right)$, followed by time propagation on the classical potential energy surface, $V\left(s_{c}, \mathbf{Q}_{\mathbf{c}}\right)$. The reaction rate constant evaluated via this approximation will be denoted $k_{\mathrm{CeS}}$ (the subscript CeS stands for Centroid Sampling),

$$
k_{\mathrm{CeS}}=-\frac{\int d s_{c} \int d p_{c} \int d \mathbf{Q}_{\mathbf{c}} \int d \mathbf{P}_{\mathbf{c}} \boldsymbol{\rho}_{c}^{\prime}\left(s_{c}, \mathbf{Q}_{\mathbf{c}}\right) e^{-\beta\left[\Sigma_{i=1, N}\left[\left(P_{c}^{(i)}\right)^{2} / 2 M^{(i)}\right]+\left(p_{c}^{2} / 2 m\right)\right]} s_{c}^{(\mathrm{Cl})}(-t) \frac{p_{c}}{m}}{\int d s_{c} \int d p_{c} \int d \mathbf{Q}_{\mathbf{c}} \int d \mathbf{P}_{\mathbf{c}} \rho_{c}\left(s_{c}, p_{c}, \mathbf{Q}_{\mathbf{c}}, \mathbf{P}_{\mathbf{c}}\right) \delta s_{c} \delta h_{c}\left(s_{c}, \mathbf{Q}_{\mathbf{c}}\right)} .
$$




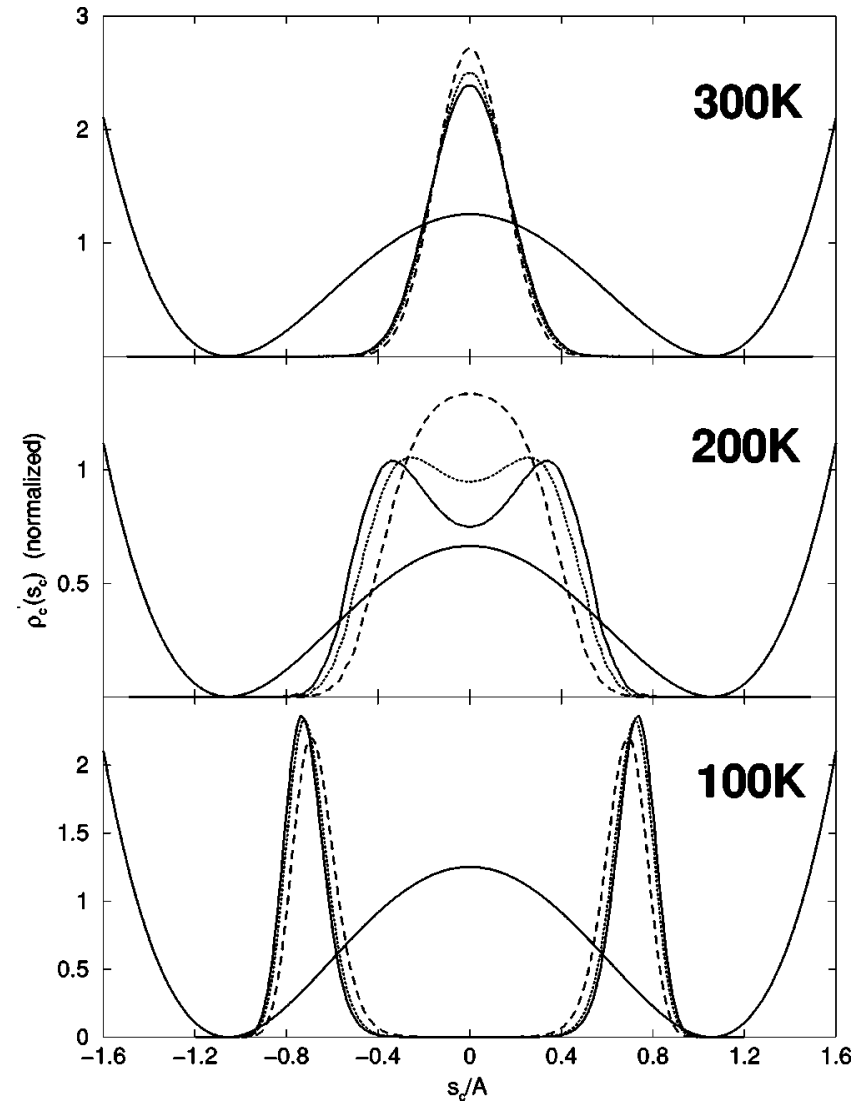

FIG. 4. The initial constrained centroid distribution $\rho_{c}^{\prime}\left(s_{c}\right)$ (normalized), for DW1 at $300 \mathrm{~K}, 200 \mathrm{~K}$, and $100 \mathrm{~K}$. The distribution is shown for various values of $\eta /\left(m \omega_{b}\right): 0.047$ (solid), 0.942 (dotted), and 2.825 (dashed). The classical potential along the reaction coordinate is also shown for reference.

It should be emphasized that Eq. (26) differs from the original CMD rate constant, Eq. (8), in that $s_{c}^{(\mathrm{Cl})}(-t)$ is propagated on the classical potential energy surface, $V\left(s_{c}, \mathbf{Q}_{\mathbf{c}}\right)$, rather than the centroid potential energy surface, $V_{c m}\left(s_{c}, \mathbf{Q}_{\mathbf{c}}\right)$.

The transmission coefficients obtained via the CeS approximation at $300 \mathrm{~K}, 200 \mathrm{~K}$, and $100 \mathrm{~K}$ are presented by dashed lines in Figs. 1, 2, and 3, respectively, for a wide range of frictions. The corresponding (normalized) constrained centroid distributions, $\rho_{c}^{\prime}\left(s_{c}\right)$, are shown in Fig. 4 (these figures were already included in Ref. 36 and are presented here for the sake of completeness). At the relatively high temperature of $300 \mathrm{~K}$, the distribution is found to be unimodal and fairly localized around the barrier top, and increasing the friction further localizes it. A somewhat different picture emerges at $200 \mathrm{~K}$ : At high frictions, the distribution is wider than at $300 \mathrm{~K}$ but still unimodal and localized around the barrier top; At low frictions, the distribution becomes bimodal. This is because lowering the temperature and/or friction leads to more extended chains. One of the beads has to be attached to the barrier top, but the rest of the beads seek regions of lower potential energy which are downhill on both sides of the barrier. As a result, the corresponding centroid distribution acquires a symmetric bimodal structure. This behavior is further enhanced at $100 \mathrm{~K}$, where $\rho_{c}^{\prime}\left(s_{c}\right)$ is seen to consist of two, clearly separated peaks on both sides of the barrier.

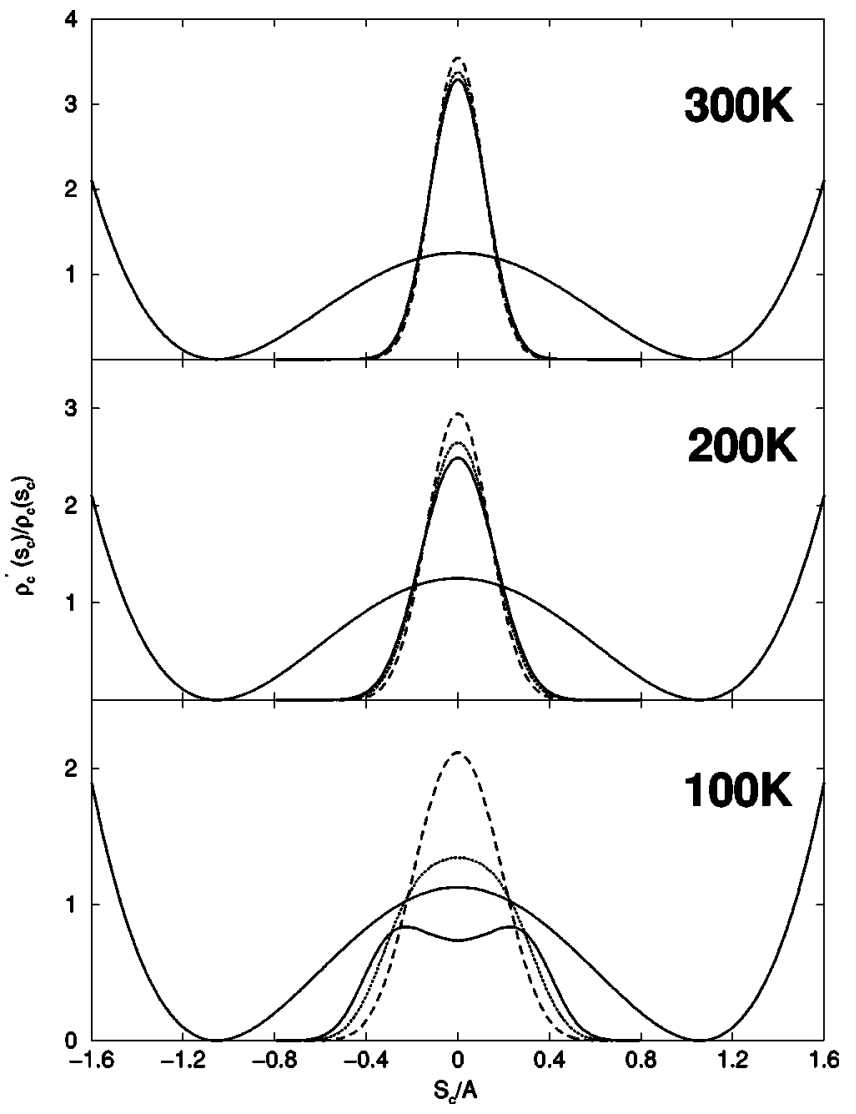

FIG. 5. $\rho_{c}^{\prime}\left(s_{c}\right) / \rho_{c}\left(s_{c}\right)$ as a function of $s_{c}$ at $300 \mathrm{~K}, 200 \mathrm{~K}$, and $100 \mathrm{~K}$. The distributions are shown for different values of $\eta /\left(m \omega_{b}\right): 0.047$ (solid), 0.942 (dotted), and 2.825 (dashed). The classical potential along the reaction coordinate is also shown for reference. It should be noted that $\rho_{c}^{\prime}\left(s_{c}\right) / \rho_{c}\left(s_{c}\right)$ decays to zero before reaching the potential minima.

Figures 1, 2, and 3 clearly indicate that centroid sampling plays a much bigger role than centroid dynamics in reproducing the quantum enhancement of the rate constants. In fact, large deviations between $k_{\mathrm{CMD}}$ and $k_{\mathrm{CeS}}$ only appear at $100 \mathrm{~K}$, which is the lowest temperature considered, at small to intermediate frictions. More importantly, the full $\mathrm{CMD}$ and the $\mathrm{CeS}$ calculations coincide at high frictions, for all temperatures considered, which is the relevant region for many condensed phase systems. Hence, at least for the model studied, combining centroid sampling with classical dynamics of the centroid is an excellent approximation that may provide significant saving in computational effort.

It should be noted that starting all the trajectories at the barrier top and propagating them in time on the classical surface would lead to the classical rate constant, which is known to be a very poor approximation. ${ }^{36}$ Thus, the above finding implies that sampling the initial states from the centroid distribution instead of starting them at the barrier top can compensate, to a large extent, for this discrepancy. Comparing the $\mathrm{CMD}$ and $\mathrm{CeS}$ approximations, we see that a large portion of the quantum enhancement is achieved by sampling the initial states with corrected statistical weights. However, the CeS approximation cannot capture dynamical tunneling effects that are sensitive to the effective barrier height, and as a results is expected to deteriorate, in general, 
at low temperatures and frictions. These expectations are confirmed by the results in Figs. 1, 2, and 3.

The similarity of the CeS approximation to the linearized version of the semiclassical approximation ${ }^{11,25,57}$ is also worth noting. In this case, the semiclassical approximation amounts to doing the initial sampling based on the Wigner distribution of the combined Boltzmann and flux operators, followed by fully classical dynamics. The above approximation is similar to the linearized semiclassical approximation, in the sense that quantum corrections are introduced via a nonclassical initial sampling. However, despite the similar spirit of the two approaches, the additional important differences should be highlighted: (1) The initial centroid distribution function is fundamentally different from the Wigner distribution, ${ }^{42}$ and (2) Calculating the centroid distribution for realistic systems is feasible, while calculating the Wigner distribution for realistic systems may be extremely difficult (however, see, for example, Refs. 58 and 59 for recent progress in this area).

\section{CLASSICAL-LIKE SAMPLING AND CENTROID DYNAMICS}

The delocalized nature of the initial distribution of centroid positions makes it increasingly more difficult to calculate $k_{\mathrm{CMD}}$ and $k_{\mathrm{CeS}}$ at very low temperatures. As the temperature decreases, the initial distribution acquires a bimodal shape with sharp picks on both sides of, and far away from, the barrier top (cf. Fig. 4). This distribution results in a situation where the large majority of the trajectories start far from the barrier top and therefore have a very small likelihood of crossing the barrier. At the same time, a small minority of the trajectories, which start in the close vicinity of the barrier top, are very likely to cross the barrier. As it turns out, the two types of trajectories make comparable contributions to the rate constant (the low likelihood of crossing the barrier is compensated for by the high probability of starting far from the barrier top, and vice versa). Efficient sampling of the trajectories that start in the close vicinity of the barrier top is possible via umbrella sampling. However, sampling of the trajectories that start far from the barrier top is made increasingly more demanding due to the inherent rare event statistics. In other words, more and more trajectories need to be sampled in order to obtain good statistics by having enough of them cross the barrier. This is demonstrated by the relatively large error bars on the values of $k_{\mathrm{CMD}}$ and $k_{\mathrm{CeS}}$ at $T=100 \mathrm{~K}$, cf. Fig. 3, and by the fact that we were unable to calculate $k_{\mathrm{CMD}}$ and $k_{\mathrm{CeS}}$ at the lower temperatures of $75 \mathrm{~K}$ and $50 \mathrm{~K}$, with reasonable computational effort. It is therefore natural to look for another centroid-based approximation for the reaction rate constant that avoids this difficulty.

One possibility is to combine classical sampling with centroid dynamics, i.e., the following expression for the rate constant;

$$
k_{\mathrm{CIS}}^{\prime}=-\frac{\int d s_{c} \int d p_{c} \int d \mathbf{Q}_{\mathbf{c}} \int d \mathbf{P}_{\mathbf{c}} e^{-\beta V\left(s_{c}, \mathbf{Q}_{\mathbf{c}}\right)} e^{-\beta\left[\Sigma_{i=1, N}\left[\left(P_{c}^{(i)}\right)^{2} / 2 M^{(i)}\right]+\left(p_{c}^{2} / 2 m\right)\right]} s_{c}(-t) \frac{p_{c}}{m} \delta\left(s_{c}\right)}{\int d s_{c} \int d p_{c} \int d \mathbf{Q}_{\mathbf{c}} \int d \mathbf{P}_{\mathbf{c}} e^{-\beta V\left(s_{c}, \mathbf{Q}_{\mathbf{c}}\right)} e^{-\beta\left[\Sigma_{i=1, N}\left[\left(P_{c}^{(i)}\right)^{2} / 2 M^{(i)}\right]+\left(p_{c}^{2} / 2 m\right)\right]} \delta s_{c} \delta h\left(s_{c}\right)},
$$

where $s_{c}(-t)$ is assumed to be propagated on the centroid potential, [the subscript ClS in Eq. (27) stands for classical sampling]. However, our conclusion from Sec. III that centroid sampling plays a more important role than centroid dynamics also implies that this would be a poor approximation. Nevertheless, it is important to note that the classical sampling in Eq. (27) consists of two, and distinctly different, approximations:

(1) The centroid symbols of the flux and heaviside operators, Eq. (10), are replaced by the corresponding classical-like approximations, $\left[p_{c} / m\right] \delta\left(s_{c}\right)$ and $h\left(s_{c}\right)$, respectively.
(2) The initial centroid distribution, $e^{-\beta V_{c m}\left(s_{c}, \mathbf{Q}_{\mathbf{c}}\right) /}$ $\int d s_{c} \int d \mathbf{Q}_{\mathbf{c}} e^{-\beta V_{c m}\left(s_{c}, \mathbf{Q}_{\mathbf{c}}\right)}$, is replaced by the classical distribution, $e^{-\beta V\left(s_{c}, \mathbf{Q}_{\mathbf{c}}\right)} / \int d s_{c} \int d \mathbf{Q}_{\mathbf{c}} e^{-\beta V\left(s_{c}, \mathbf{Q}_{\mathbf{c}}\right)}$.

One may now wonder if an improved approximation can emerge from avoiding one of these approximations. A particularly appealing possibility is to avoid the second approximation, which would amount to replacing the classical potential, $V\left(s_{c}, \mathbf{Q}_{\mathbf{c}}\right)$, in Eq. (27) with the centroid potential, $V_{c m}\left(s_{c}, \mathbf{Q}_{\mathbf{c}}\right)$. This would lead to an expression for the reaction rate constant which is identical to that in a fictitious classical system that moves on the centroid potential,

$$
k_{\mathrm{CIS}}=-\frac{\int d s_{c} \int d p_{c} \int d \mathbf{Q}_{\mathbf{c}} \int d \mathbf{P}_{\mathbf{c}} e^{-\beta V_{c m}\left(s_{c}, \mathbf{Q}_{\mathbf{c}}\right)} e^{-\beta\left[\Sigma_{i=1, N}\left(P_{c}^{(i)}\right)^{2} / 2 M^{(i)}+p_{c}^{2} / 2 m\right]} s_{c}(-t) \frac{p_{c}}{m} \delta\left(s_{c}\right)}{\int d s_{c} \int d p_{c} \int d \mathbf{Q}_{\mathbf{c}} \int d \mathbf{P}_{\mathbf{c}} e^{-\beta V_{c m}\left(s_{c}, \mathbf{Q}_{\mathbf{c}}\right)} e^{-\beta\left[\Sigma_{i=1, N}\left(P_{c}^{(i)}\right)^{2} / 2 M^{(i)}+p_{c}^{2} / 2 m\right]} \delta s_{c} \delta h\left(s_{c}\right)} .
$$


As was shown in Ref. 36, Eq. (28) is one of a family of possible completely equivalent expressions for the classical rate constant. The origin of this variety has to do with the fact that the rate constant is independent of the initial perturbation that shifts the reactive system from equilibrium. A perturbation linear in $s$ wouldlead, via linear response theory, to an expression for the classical rate constant in terms of a position-flux correlation function, such as Eq. (28). ${ }^{36}$ A perturbation linear in $h(s)$ would lead to an expression for the classical rate constant in terms of a heaviside-flux correlation function,

$$
k_{\mathrm{CIS}}=\frac{\int d s_{c} \int d p_{c} \int d \mathbf{Q}_{\mathbf{c}} \int d \mathbf{P}_{\mathbf{c}} e^{-\beta\left[\Sigma_{i=1, N}\left[\left(P_{c}^{(i)}\right)^{2} / 2 M^{(i)}\right]+\left(p_{c}^{2} / 2 m\right)+V_{c m}\left(s_{c}, \mathbf{Q}_{\mathbf{c}}\right)\right.} h\left[s_{c}(t)\right] \frac{p_{c}}{m} \delta\left(s_{c}\right)}{\int d s_{c} \int d p_{c} \int d \mathbf{Q}_{\mathbf{c}} \int d \mathbf{P}_{\mathbf{c}} e^{-\beta\left[\Sigma_{i=1, N}\left[\left(P_{c}^{(i)}\right)^{2} / 2 M^{(i)}\right]+\left(p_{c}^{2} / 2 m\right)+V_{c m}\left(s_{c}, \mathbf{Q}_{\mathbf{c}}\right)\right]}\left[\delta h\left(s_{c}\right)\right]^{2}} .
$$

It is crucial to note that although Eqs. (28) and (29) appear to be different from each other, they are bound to give the same value of $k_{\mathrm{ClS}} \cdot{ }^{36}$

It should be noted that Eq. (29) has been previously proposed by Schenter et al., as a way of introducing dynamical corrections to PI-QTST. ${ }^{20}$ Going in reverse, one can now reproduce PI-QTST from Eq. (29) by taking its TST limit,

$$
k_{\mathrm{CIS}} \approx k_{\mathrm{CIS}}^{\mathrm{TST}}=\frac{\int d s_{c} \int d p_{c} \int d \mathbf{Q}_{\mathbf{c}} \int d \mathbf{P}_{\mathbf{c}} e^{-\beta\left[\Sigma_{i=1, N}\left[\left(P_{c}^{(i)}\right)^{2} / 2 M^{(i)}\right]+\left(p_{c}^{2} / 2 m\right)+V_{c m}\left(s_{c}, \mathbf{Q}_{\mathbf{c}}\right)\right]} h\left[p_{c}\right] \frac{p_{c}}{m} \delta\left(s_{c}\right)}{\int d s_{c} \int d p_{c} \int d \mathbf{Q}_{\mathbf{c}} \int d \mathbf{P}_{\mathbf{c}} e^{-\beta\left[\Sigma_{i=1, N}\left[\left(P_{c}^{(i)}\right)^{2} / 2 M^{(i)}\right]+\left(p_{c}^{2} / 2 m\right)+V_{c m}\left(s_{c}, \mathbf{Q}_{\mathbf{c}}\right)\right.}\left[\delta h\left(s_{c}\right)\right]^{2}} .
$$

In fact, $k_{\mathrm{CIS}}^{\mathrm{TST}}$ corresponds to a primitive version of the PI-QTST rate constant, which upon variational optimization turns out to be a rather good approximation at intermediate to strong frictions. ${ }^{6,8,9,36,44}$ Thus, the approximation embodied in Eq. (29) naturally relates CMD with PI-QTST for a multidimensional system.

An illuminating argument as to why and when one can expect the approximation in Eq. (29) or Eq. (28) to work can be presented in the case of a system bilinearly coupled to a harmonic bath. The analysis is based on the fact that in this case $\rho_{c}^{\prime}\left(s_{c}, \mathbf{Q}_{\mathbf{c}}\right) / \rho_{c}\left(s_{c}, \mathbf{Q}_{\mathbf{c}}\right)$ is independent of $\mathbf{Q}_{\mathbf{c}}(\mathrm{cf}$. Sec. II B),

$$
\frac{\rho_{c}^{\prime}\left(s_{c}, \mathbf{Q}_{\mathbf{c}}\right)}{\rho_{c}\left(s_{c}, \mathbf{Q}_{\mathbf{c}}\right)}=\rho_{1}\left(s_{c}\right)
$$

As a result, one can approximate $k_{\mathrm{CMD}}$ in the following way:

$$
k_{\mathrm{CMD}} \approx \int d s_{c} \rho_{1}\left(s_{c}\right) k^{\prime}\left(s_{c}\right),
$$

where

$$
k^{\prime}\left(s_{c}\right)=-\frac{\int d s_{c}^{\prime} \int d p_{c} \int d \mathbf{Q}_{c} \int d \mathbf{P}_{\mathbf{c}} e^{-\beta\left[\Sigma_{i=1, N}\left[\left(P_{c}^{(i)}\right)^{2} / 2 M^{(i)}\right]+\left(p_{c}^{2} / 2 m\right)+V_{c m}\left(s_{c}^{\prime}, \mathbf{Q}_{\mathbf{c}}\right) s_{c}^{\prime}(-t) \frac{p_{c}}{m} \delta\left(s_{c}^{\prime}-s_{c}\right)\right.}}{\int d s_{c}^{\prime} \int d p_{c} \int d \mathbf{Q}_{\mathbf{c}} \int d \mathbf{P}_{\mathbf{c}} e^{-\beta\left[\Sigma_{i=1, N}\left[\left(P_{c}^{(i)}\right)^{2} / 2 M^{(i)}\right]+\left(p_{c}^{2} / 2 m\right)+V_{c m}\left(s_{c}^{\prime}, \mathbf{Q}_{c}\right)\right]} \delta s_{c}^{\prime} \delta h\left(s_{c}^{\prime}\right)} .
$$

The only approximation in Eq. (32) involves replacing $h_{c}\left(s_{c}\right)$ by $h\left(s_{c}\right)$ in the denominator of Eq. (33), which is expected to be generally valid. It should also be noted that $s_{c}$ in the denominator was renamed and is now denoted by $s_{c}^{\prime}$.

We now note that $\rho_{c}\left(s_{c}\right)$ is concentrated in the close vicinity of the bottoms of the product and reactant wells. Hence, $\delta h\left(s_{c}^{\prime}\right)$ can be replaced by $\delta h\left(s_{c}^{\prime}-s_{c}^{*}\right)$, as long as $s_{c}^{*}$ does not extend to areas in the close vicinity of the bottoms of these wells. In particular, $\delta h\left(s_{c}^{\prime}\right)$ may be substituted by $\delta h\left(s_{c}^{\prime}-s_{c}\right)$ as long as $\rho_{1}\left(s_{c}\right)$ decays to zero before reaching the minima of the potential. Figure 5 demonstrates that this is a valid assumption for a wide range of temperatures and frictions.

The above assumptions lead to the following expression for $k^{\prime}\left(s_{c}\right)$ :

$$
k^{\prime}\left(s_{c}\right) \approx-\frac{\int d s_{c}^{\prime} \int d p_{c} \int d \mathbf{Q}_{\mathbf{c}} \int d \mathbf{P}_{\mathbf{c}} e^{-\beta\left[\Sigma_{i=1, N}\left[\left(P_{c}^{(i)}\right)^{2} / 2 M^{(i)}\right]+\left(p_{c}^{2} / 2 m\right)+V_{c m}\left(s_{c}^{\prime}, \mathbf{Q}_{\mathbf{c}}\right)\right]} s_{c}^{\prime}(-t) \frac{p_{c}}{m} \delta\left(s_{c}^{\prime}-s_{c}\right)}{\int d s_{c}^{\prime} \int d p_{c} \int d \mathbf{Q}_{\mathbf{c}} \int d \mathbf{P}_{\mathbf{c}} e^{-\beta\left[\Sigma_{i=1, N}\left[\left(P_{c}^{(i)}\right)^{2} / 2 M^{(i)}\right]+\left(p_{c}^{2} / 2 m\right)+V_{c m}\left(s_{c}^{\prime}, \mathbf{Q}_{\mathbf{c}}\right)\right]} \delta s_{c}^{\prime} \delta h_{c}\left(s_{c}^{\prime}-s_{c}\right)} .
$$


Thus, if we now think of $s_{c}^{\prime}$ as the position variable, and of $s_{c}$ as a parameter, then $k^{\prime}\left(s_{c}\right)$ can be interpreted as a classical-like rate constant, calculated on the centroid potential via the reactive flux method, with the dividing surface set at $s_{c}^{\prime}=s_{c}$. Since the rate constant is expected to be independent of the location of the dividing surface, as long as $s_{c}$ is not in the close vicinity of the potential minima, we can replace $k^{\prime}\left(s_{c}\right)$ by $k^{\prime}\left(s_{c}=0\right) \equiv k_{\mathrm{ClS}}$. This leads to our final result, namely,

$$
k_{\mathrm{CMD}} \approx k_{\mathrm{ClS}}^{K} \equiv K \times k_{\mathrm{ClS}},
$$

where

$$
K=\int d s_{c} \rho_{1}\left(s_{c}\right)=\int d s_{c} \frac{\rho_{c}^{\prime}\left(s_{c}, \mathbf{Q}_{\mathbf{c}}\right)}{\rho_{c}\left(s_{c}, \mathbf{Q}_{\mathbf{c}}\right)} .
$$

It is interesting to note that $K$ in Eq. (36) coincides with the "CMD transmission coefficient" as defined by Jang and Voth in Ref. 12. However, the analysis in Ref. 12 differs from the one presented here in two important respects:

(1) The discussion in Ref. 12 is restricted to a onedimensional system (the reaction coordinate), and does not include coupling to a (harmonic) bath.

(2) The potential along the reaction coordinate in Ref. 12 is assumed to be unbounded at $x= \pm \infty$, as opposed to the double-well shape assumed here. It should be noted that one cannot define a rate constant for a one-dimensional system unless the potential is not bounded, and that in the case of an unbounded one-dimensional potential, the classical TST rate constant coincides with the exact classical rate constant.

Thus, Eq. (36) provides an extension of the corresponding result in Ref. 12, to cases involving bounded reactive potentials and coupling to a harmonic bath.

Equation (35) would coincide with Eq. (29) if $K=1$. This is certainly true in the classical limit, where $\rho_{1}\left(s_{c}\right)$ $=\delta\left(s_{c}\right)$. Figure 6 shows that the value of $K$ remains very close to 1 for the model system considered, on a wide range of temperatures and frictions, and that the main deviations occur at very low temperatures and frictions. Hence, $k_{\mathrm{ClS}}$ is expected to provide a very good approximation for $k_{\mathrm{CMD}}$, at least in the case of a system bilinearly coupled to a harmonic bath.

Although it is difficult to generalize the above derivation

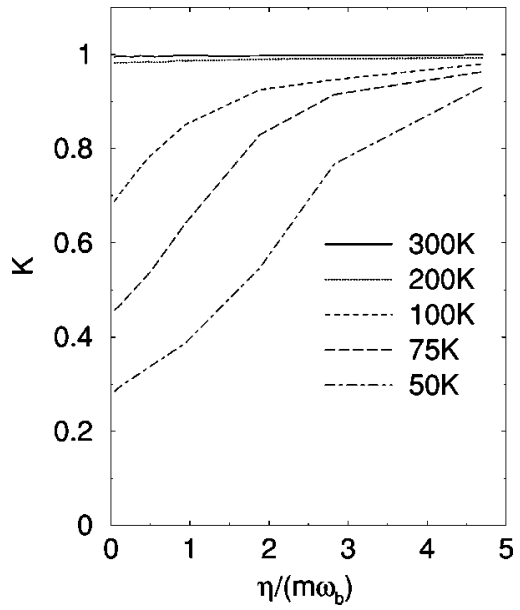

FIG. 6. $K$ as a function of friction at various temperatures.

to the case of a general anharmonic system, it seems reasonable to expect that $k_{\mathrm{CIS}}$ will also provide a useful approximation in such situations. In some cases, it may also be possible to formulate feasible approximations for the effective correction factor, $K$.

$k_{\mathrm{CIS}}$ was calculated numerically for a symmetric doublewell bilinearly coupled to a harmonic bath and the results are represented by the dotted lines in Figs. 1, 2, and 3 for a wide range of temperatures and frictions. From these figures it is clear that Eq. (29) provides an excellent approximation to $k_{\mathrm{CMD}}$. In fact, $k_{\mathrm{CIS}}$ turn out to provide a somewhat better approximation than $k_{\mathrm{CMD}}$ when compared to the exact results. Surprisingly, $k_{\mathrm{CIS}}$ also provides a slightly better approximation in comparison to $k_{\mathrm{ClS}}^{K}$, since $K$ is generally smaller than one (cf. Fig. 6).

The calculation of $k_{\mathrm{CIS}}$ in the general case will require an on-the-fly evaluation of the centroid potential, and will therefore still be far more computationally demanding in comparison to the calculation of $k_{\mathrm{CeS}}$. PI-QTST, which was shown above to be an approximated version of $k_{\mathrm{CIS}}$, provides one attractive way of reducing the computational effort involved in evaluating $k_{\mathrm{CIS}}$, but cannot directly account for dynamical effects. Another interesting simplification which was originally suggested by Schenter et al., amounts to performing the dynamics on the classical potential. ${ }^{20}$ Applying this approximation to Eq. (28) leads to the following simplified expression for the reaction rate constant:

$$
k_{\mathrm{CIS}}^{c}=-\frac{\int d s_{c} \int d p_{c} \int d \mathbf{Q}_{\mathbf{c}} \int d \mathbf{P}_{\mathbf{c}} e^{-\beta V_{c m}\left(s_{c}, \mathbf{Q}_{\mathbf{c}}\right)} e^{-\beta\left[\Sigma_{i=1, N}\left[\left(P_{c}^{(i)}\right)^{2} / 2 M^{(i)}\right]+\left(p_{c}^{2} / 2 m\right)\right]} s_{c}^{(\mathrm{Cl})}(-t) \frac{p_{c}}{m} \delta\left(s_{c}\right)}{\int d s_{c} \int d p_{c} \int d \mathbf{Q}_{\mathbf{c}} \int d \mathbf{P}_{\mathbf{c}} e^{-\beta V_{c m}\left(s_{c}, \mathbf{Q}_{\mathbf{c}}\right)} e^{-\beta\left[\Sigma_{i=1, N}\left[\left(P_{c}^{(i)}\right)^{2} / 2 M^{(i)}\right]+\left(p_{c}^{2} / 2 m\right)\right]} \delta s_{c} \delta h\left(s_{c}\right)} .
$$

Propagating on the classical potential with its higher barrier is expected to lead to less recrossing, and hence to larger reaction rate constants. Hence, one expects $k_{\mathrm{CIS}}^{c}$ to be larger than $k_{\mathrm{ClS}}$. At the same time, $k_{\mathrm{CIS}}$ is not expected to account to the full extent for the quantum enhancement of the rate constant, and hence $k_{\mathrm{ClS}}$ is expected to be smaller than the exact rate constant (cf. Figs. 1, 2, and 3). Hence, $k_{\mathrm{CIS}}^{c}$ may actually turn out to provide a better estimate of the reaction rate constant! This indeed turns out to be the case for our model system (cf. the solid lines with opaque squares in 


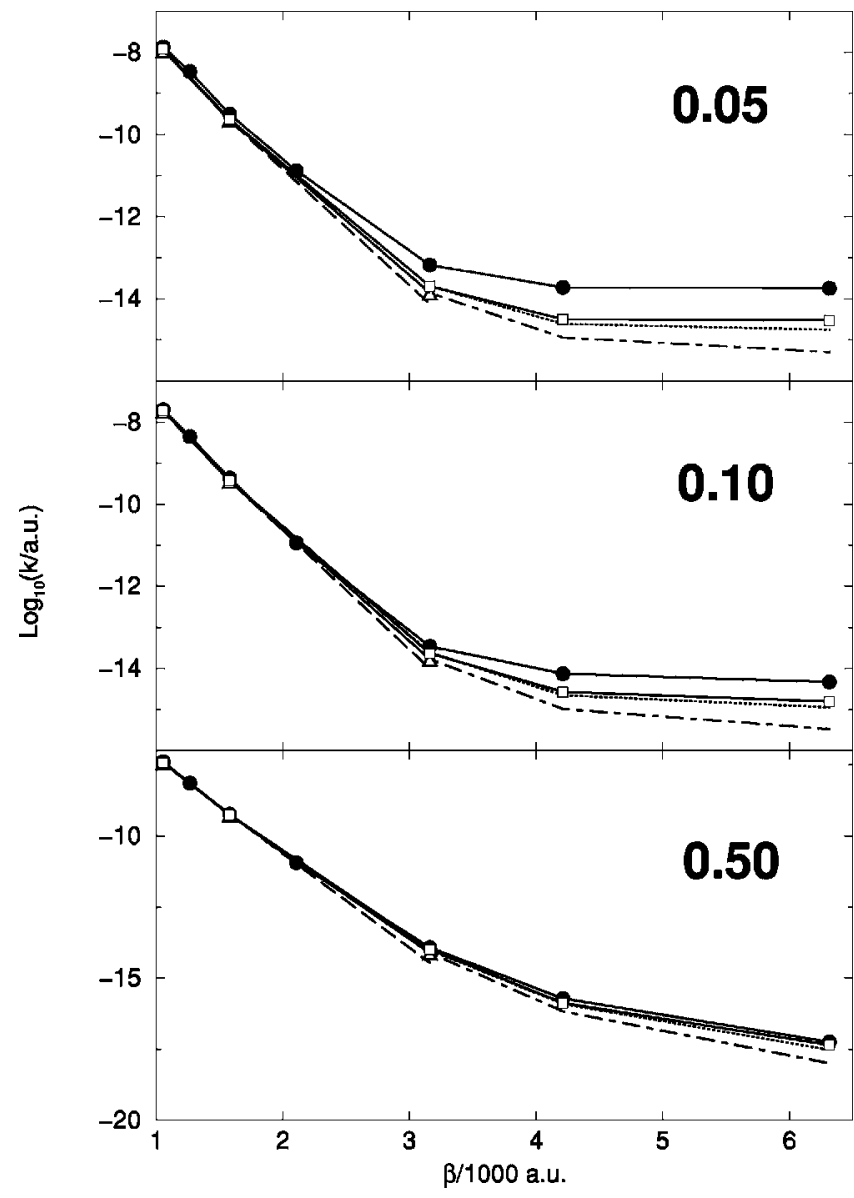

FIG. 7. $\log _{10}(k /$ a.u. $)$ as a function of inverse temperature, at three different frictions: $\eta /\left(m \omega_{b}\right)=0.05,0.1,0.5$. The results obtained by different methods are given at each temperature: exact $k$ (solid line with filled circles), $k_{\mathrm{CMD}}$ (solid line with opaque triangles), $k_{\mathrm{ClS}}^{K}$ (dotted-dashed line), $k_{\mathrm{CIS}}$ (dotted line), $k_{\mathrm{CeS}}$ (dashed line), and $k_{\mathrm{CIS}}^{c}$ (solid line with opaque squares).

Figs. 1, 2, and 3). Despite this, one should not lose sight of the fact that this better agreement is the result of a rather fortunate cancellation of errors: The enhancement of the exact rate constant relative to $k_{\mathrm{ClS}}$ is probably due to the inability of the latter to fully account for quantum tunneling, while the enhancement of $k_{\mathrm{CIS}}^{c}$ relative to $k_{\mathrm{CIS}}$ originates from diminished classical-like recrossing.

Finally, it should be noted that the calculation of $k_{\mathrm{CIS}}$, $k_{\mathrm{CIS}}^{K}$, and $k_{\mathrm{CIS}}^{c}$ involves initial trajectories that start at the barrier top. Hence, unlike the calculation of $k_{\mathrm{CMD}}$ and $k_{\mathrm{CeS}}$, the calculation of $k_{\mathrm{CIS}}, k_{\mathrm{CIS}}^{K}$, and $k_{\mathrm{CIS}}^{c}$ can be easily extended to very low temperatures. In Fig. 7, we present the exact rate constants (on a logarithmic scale), as well as the various approximations discussed in this paper $\left(k_{\mathrm{CMD}}, k_{\mathrm{ClS}}^{K}, k_{\mathrm{ClS}}\right.$, $k_{\mathrm{CeS}}$, and $k_{\mathrm{CIS}}^{c}$ ), as a function of the inverse temperature. Special attention should be given to the two lowest temperatures considered $(75 \mathrm{~K}$ and $50 \mathrm{~K})$. We were unable to calculate $k_{\mathrm{CMD}}$ and $k_{\mathrm{CeS}}$ at these temperatures with reasonable computational effort, due to the rare event statistics mentioned in the beginning of this section. ${ }^{36}$ The approximations represented by $k_{\mathrm{CIS}}, k_{\mathrm{CIS}}^{K}$, and $k_{\mathrm{CIS}}^{c}$ avoid this difficulty by starting all trajectories at the barrier top. As a result, $k_{\mathrm{CIS}}$, $k_{\mathrm{CIS}}^{K}$, and $k_{\mathrm{CIS}}^{c}$ could be calculated with ease at very low temperatures, and the agreement with the exact $k$ is found to be rather good, especially at the high friction region which is relevant to many condensed phase systems.

\section{CONCLUDING REMARKS}

Two approximations to the CMD reaction rate constant, $k_{\mathrm{CMD}}$, have been considered. The first approximation, which was denoted by $k_{\mathrm{CeS}}$, combines centroid sampling of the initial state with classical dynamics. Its most attractive feature is that it only requires one PIMD/PIMC simulation at the initial time, thereby providing an economical centroidbased route for calculating quantum mechanical reaction rate constants. Its main disadvantage is that dynamical quantum effects that are not included in the initial nonclassical sampling, are not accounted for. Another disadvantage has to do with the delocalized nature of the initial centroid distribution, that can lead to inefficient sampling at very low temperatures. The second approximation, which was denoted by $k_{\mathrm{CIS}}$, correspond to a classical-like calculation of the reaction rate constant, on the centroid potential. It was shown that this approximation can be rigorously justified when the bath is harmonic. Its main advantage is that, unlike in the calculation of $k_{\mathrm{CMD}}$, all the trajectories start at the barrier top. Thus, the problem of poor sampling due to the highly delocalized nature of the initial centroid distribution does not arise, and the calculation of rate constants at very low temperatures becomes possible. The main disadvantage of this approximation is that, similarly to $k_{\mathrm{CMD}}$, the calculation of $k_{\mathrm{CIS}}$ requires that the dynamics is carried out on the centroid potential. Hence, in the general case, the centroid force has to be calculated on-the-fly, which would require performing a PIMD/PIMC calculation at every time step. A reduction of the computational effort involved can be achieved by applying a TST-like approximation to $k_{\mathrm{CIS}}$, which leads to PIQTST, or by performing the dynamics classically, which leads to the approximation embodied in $k_{\mathrm{CIS}}^{c}$.

The various approximations were tested on the exactly solvable model of a symmetrical double-well bilinearly coupled to a harmonic bath, for a wide range of temperatures and frictions. $k_{\mathrm{CeS}}$ was found to be a good approximation of $k_{\mathrm{CMD}}$, except at very low temperatures and small frictions. $k_{\mathrm{CIS}}$ was typically found to provide superior agreement with $k_{\mathrm{CMD}}$, and in fact performed better than $k_{\mathrm{CMD}}$ in reproducing the exact results, which is likely to be accidental. It is important to note that the variation in the predictions of different centroid-based approximations is far smaller relative to the gap between the quantum and classical rate constants. Thus, perhaps the most important conclusion from the present work is that regardless of which centroid-based approximation is used, the result obtained is expected to provide a far better estimate to the rate constant than the corresponding classical result. This observation seem to reinforce the importance of the centroid concept as a mean for estimating quantum mechanical rate constants. At the same time, it appears that the success of the centroid based methods, at least for the benchmark considered, relies heavily on their ability to capture the quantum statics, rather than the quantum dynamics of the problem. This is demonstrated by the fact that the results are rather insensitive to whether we use classical 
dynamics or centroid dynamics. Future work will therefore focus on (1) exploring whether or not the above observations extend to more anharmonic systems; (2) testing CMD in problems that show a more pronounced signature of quantum dynamics.

\section{ACKNOWLEDGMENTS}

This work was supported by startup funding from the University of Michigan. E.G. is grateful to Professor Gregory A. Voth and Dr. Seogjoo Jang for helpful discussions and their encouragement.

${ }^{1}$ P. Hänggi, P. Talkner, and M. Borkovec, Rev. Mod. Phys. 62, 251 (1990).

${ }^{2}$ W. H. Miller, J. Chem. Phys. 61, 1823 (1974).

${ }^{3}$ M. J. Gillan, Phys. Rev. Lett. 58, 563 (1987).

${ }^{4}$ M. J. Gillan, J. Phys. C 20, 3621 (1987).

${ }^{5}$ G. A. Voth, D. Chandler, and W. H. Miller, J. Chem. Phys. 91, 7749 (1989).

${ }^{6}$ G. A. Voth, Chem. Phys. Lett. 170, 289 (1990).

${ }^{7}$ R. P. McRae, G. K. Schenter, B. C. Garrett, G. R. Haynes, G. A. Voth, and G. C. Schatz, J. Chem. Phys. 97, 7392 (1992).

${ }^{8}$ G. A. Voth, J. Phys. Chem. 97, 8365 (1993).

${ }^{9}$ G. A. Voth, Adv. Chem. Phys. 93, 135 (1996).

${ }^{10}$ N. Fisher and H. C. Andersen, J. Phys. Chem. 100, 1137 (1996).

${ }^{11}$ E. Pollak and J. Liao, J. Chem. Phys. 108, 2733 (1998).

${ }^{12}$ S. Jang and G. A. Voth, J. Chem. Phys. 112, 8747 (2000).

${ }^{13}$ J. L. Liao and E. Pollak, Chem. Phys. 268, 295 (2001).

${ }^{14}$ R. P. Feynman and A. R. Hibbs, Quantum Mechanics and Path Integrals (McGraw-Hill, New York, 1965).

${ }^{15}$ R. P. Feynman, Statistical Mechanics (Benjamin, New York, 1972).

${ }^{16}$ B. J. Berne and D. Thirumalai, Annu. Rev. Phys. Chem. 37, 401 (1986).

${ }^{17}$ D. M. Ceperley, Rev. Mod. Phys. 67, 279 (1995).

${ }^{18}$ P. Pechukas, Dynamics of Molecular Collisions, Part 2 (Plenum, New York, 1976), p. 269.

${ }^{19}$ M. Messina, G. K. Schenter, and B. C. Garrett, J. Chem. Phys. 98, 8525 (1993).

${ }^{20}$ G. K. Schenter, M. Messina, and B. C. Garrett, J. Chem. Phys. 99, 1674 (1993).

${ }^{21}$ T. Yamamoto, J. Chem. Phys. 33, 281 (1960).

${ }^{22}$ W. H. Miller, S. D. Schwartz, and J. W. Tromp, J. Chem. Phys. 79, 4889 (1983).

${ }^{23}$ N. Makri and K. Thompson, Chem. Phys. Lett. 291, 101 (1998).
${ }^{24}$ W. H. Miller, Faraday Discuss. 110, 1 (1998).

${ }^{25}$ H. Wang, X. Sun, and W. H. Miller, J. Chem. Phys. 108, 9726 (1998).

${ }^{26}$ J. S. Shao and N. Makri, J. Phys. Chem. A 103, 7753 (1999).

${ }^{27}$ K. Thompson and N. Makri, Phys. Rev. E 59, R4729 (1999).

${ }^{28}$ H. Wang, M. Thoss, and W. H. Miller, J. Chem. Phys. 112, 47 (2000).

${ }^{29}$ K. Yamashita and W. H. Miller, J. Chem. Phys. 82, 5475 (1985).

${ }^{30}$ E. Gallicchio and B. J. Berne, J. Chem. Phys. 105, 7064 (1996).

${ }^{31}$ E. Gallicchio, S. A. Egorov, and B. J. Berne, J. Chem. Phys. 109, 7745 (1998).

${ }^{32}$ S. A. Egorov, E. Gallicchio, and B. J. Berne, J. Chem. Phys. 107, 9312 (1997).

${ }^{33}$ G. Krilov and B. J. Berne, J. Chem. Phys. 111, 9147 (1999).

${ }^{34}$ E. Rabani, G. Krilov, and B. J. Berne, J. Chem. Phys. 112, 2605 (2000).

${ }^{35}$ E. Sim, G. Krilov, and B. Berne, J. Phys. Chem. A 105, 2824 (2001).

${ }^{36}$ E. Geva, Q. Shi, and G. A. Voth, J. Chem. Phys. 115, 9209 (2001).

${ }^{37}$ J. Cao and G. A. Voth, J. Chem. Phys. 100, 5093 (1994).

${ }^{38}$ J. Cao and G. A. Voth, J. Chem. Phys. 100, 5106 (1994).

${ }^{39}$ J. Cao and G. A. Voth, J. Chem. Phys. 101, 6157 (1994).

${ }^{40}$ J. Cao and G. A. Voth, J. Chem. Phys. 101, 6168 (1994).

${ }^{41}$ J. Cao and G. A. Voth, J. Chem. Phys. 101, 6184 (1994).

${ }^{42}$ S. Jang and G. A. Voth, J. Chem. Phys. 111, 2357 (1999).

${ }^{43}$ S. Jang and G. A. Voth, J. Chem. Phys. 111, 2371 (1999).

${ }^{44}$ M. Topaler and N. Makri, J. Chem. Phys. 101, 7500 (1994).

${ }^{45}$ A. Calhoun, M. Pavese, and G. A. Voth, Chem. Phys. Lett. 262, 415 (1996).

${ }^{46}$ U. W. Schmitt and G. A. Voth, J. Chem. Phys. 111, 9361 (1999).

${ }^{47}$ S. Jang, Y. Pak, and G. A. Voth, J. Phys. Chem. A 103, 10289 (1999).

${ }^{48}$ M. Pavese and G. A. Voth, Chem. Phys. Lett. 249, 231 (1996).

${ }^{49}$ K. Kinugawa, P. B. Moore, and M. L. Klein, J. Chem. Phys. 106, 1154 (1997)

${ }^{50}$ K. Kinugawa, P. B. Moore, and M. L. Klein, J. Chem. Phys. 109, 610 (1998).

${ }^{51}$ K. Kinugawa, Chem. Phys. Lett. 292, 454 (1998).

${ }^{52}$ M. Pavese, D. R. Berard, and G. A. Voth, Chem. Phys. Lett. 300, 93 (1999).

${ }^{53}$ S. Miura, S. Okazaki, and K. Kinugawa, J. Chem. Phys. 110, 4523 (1999).

${ }^{54}$ F. J. Bermejo et al., Phys. Rev. Lett. 84, 5359 (2000).

${ }^{55}$ D. Chandler, J. Chem. Phys. 68, 2959 (1978).

${ }^{56}$ J. A. Montgomrey, Jr., D. Chandler, and B. J. Berne, J. Chem. Phys. 70, 4056 (1979)

${ }^{57}$ J. S. Shao, J. L. Liao, and E. Pollak, J. Chem. Phys. 108, 9711 (1998).

${ }^{58}$ M. Ovchinnikov, V. A. Apkarian, and G. A. Voth, J. Chem. Phys. 184, 7130 (2001).

${ }^{59}$ Y. Zheng and E. Pollak, J. Chem. Phys. 114, 9741 (2001). 